\title{
Different intermittency for longitudinal and transversal turbulent fluctuations
}

\author{
Siegfried Grossmann, Detlef Lohse, and Achim Reeh \\ Fachbereich Physik der Universität Marburg, Renthof 6, D-35032 Marburg, Germany
}

(Received 20 March 1997; accepted 2 September 1997)

\begin{abstract}
Scaling exponents of the longitudinal and transversal velocity structure functions in numerical Navier-Stokes turbulence simulations with Taylor-Reynolds numbers up to $R e_{\lambda}=110$ are determined by the extended self similarity method. We find significant differences in the degree of intermittency: For the sixth moments the scaling corrections to the classical Kolmogorov expectations are $\delta \xi_{6}^{L}=-0.21 \pm 0.01$ and $\delta \xi_{6}^{T}=-0.43 \pm 0.01$, respectively, independent of $R e_{\lambda}$. Also the generalized extended self similarity exponents $\rho_{p, q}=\delta \xi_{p} / \delta \xi_{q}$ differ significantly for the longitudinal and transversal structure functions. Within the She-Leveque model this means that longitudinal and transversal fluctuations obey different types of hierarchies of the moments. Moreover, the She-Leveque model hierarchy parameters $\beta^{L}$ and $\beta^{T}$ show small but significant dependences on the order of the moment. (C) 1997 American Institute of Physics. [S1070-6631(97)03412-0]
\end{abstract}

\section{INTRODUCTION}

One of the central issues in turbulence theory has always been whether the velocity structure functions deviate from Kolmogorov's classical expectation. ${ }^{1,2}$ For many years the community focused on the longitudinal structure function,

$$
D_{p}^{L}(r)=\left\langle\left[(\boldsymbol{u}(\boldsymbol{x}+\boldsymbol{r})-\boldsymbol{u}(\boldsymbol{x})) \cdot \boldsymbol{e}_{r}^{L}\right]^{p}\right\rangle=\left\langle\left(v^{L}(r)\right)^{p}\right\rangle,
$$

whose inertial subrange (ISR) scaling exponents we define as $\zeta_{p}^{L}$. (Here, $\boldsymbol{e}_{r}^{L}$ is the unit vector in the $\boldsymbol{r}$ direction.) The reason for this was that in many experiments Taylor's frozen flow hypothesis ${ }^{1-3}$ had to be employed and therefore, the transversal structure functions,

$$
D_{p}^{T}(r)=\left\langle\left[(\boldsymbol{u}(\boldsymbol{x}+\boldsymbol{r})-\boldsymbol{u}(\boldsymbol{x})) \cdot \boldsymbol{e}_{r}^{T}\right]^{p}\right\rangle=\left\langle\left(v^{T}(r)\right)^{p}\right\rangle,
$$

$\boldsymbol{e}_{r}^{T}$ being a unit vector perpendicular to $\boldsymbol{r}$, could not be obtained. We denote the scaling exponents of $D_{p}^{T}(r)$ as $\zeta_{p}^{T}$.

A priori, there is no reason to expect $\zeta_{p}^{L}=\zeta_{p}^{T}$ for general $p$. The probability density function (PDF) of the longitudinal velocity difference $v^{L}(r)$ is skewed because information from $\boldsymbol{x}$ to $\boldsymbol{x}+\boldsymbol{r}$ is conveyed by the velocity difference itself, and odd moments of $v^{L}(r)$ thus do not vanish, $D_{p}^{L} \neq 0$. In particular, for homogeneous and isotropic turbulence the third order longitudinal structure function is connected to the second order one by the Howard-von Karman-Kolmogorov structure equation, ${ }^{1,2}$

$$
D_{3}^{L}(r)=-\frac{4}{5} \epsilon r+6 \nu \frac{d}{d r} D_{2}^{L}(r) .
$$

Here, $\epsilon$ is the mean energy dissipation rate and $\nu$ is the kinematic viscosity. The PDF of the transversal velocity difference $v^{T}(r)$, on the other hand, is symmetric and consequently all odd order moments vanish, $D_{p}^{T}(r)=0, p$ odd. Only the second order longitudinal and transversal structure functions are expected to scale the same way ${ }^{1,4,5}$ in the large Reynolds number limit since for isotropic flow incompressibility implies ${ }^{1}$

$$
D_{2}^{T}(r)=D_{2}^{L}(r)+\frac{r}{2} \frac{d}{d r} D_{2}^{L}(r) .
$$

Recently, multi-probe and optical techniques made the transversal structure functions experimentally accessible. ${ }^{4,6-10}$ In addition, also recent numerical simulations of decaying turbulence ${ }^{11}$ have focused on the difference in scaling of $D_{p}^{L}(r)$ and $D_{p}^{T}(r)$.

The results of all this work seem to be contradictory. While all authors agree that (i) both $\zeta_{p}^{L}$ and $\zeta_{p}^{T}$ show significant deviations from the classical expectation $p / 3$, and that (ii) $\zeta_{p}^{L}$ is well fitted by the She-Leveque model (" $\mathrm{SL}$ model"), ${ }^{12}$ saying that

$$
\zeta_{p}=\frac{p}{3}-C_{0}\left(\frac{p}{3}\left(1-\beta^{3}\right)-\left(1-\beta^{p}\right)\right),
$$

with $C_{0}=2$ and $\beta=(2 / 3)^{1 / 3}$, they disagree on whether $\zeta_{p}^{L}=\zeta_{p}^{T}$ or $\zeta_{p}^{L} \neq \zeta_{p}^{T}$.

van de Water's group ${ }^{7,8}$ finds that in shear flow the (moduli of the) transversal intermittency corrections $\delta \zeta_{p}^{T}=\zeta_{p}^{T}-p / 3$ are significantly larger than the longitudinal ones $\delta \zeta_{p}^{L}=\zeta_{p}^{L}-p / 3, p>3$. The same is found for jet flow turbulence by Camussi and Benzi ${ }^{10}$ and for decaying (numerical) turbulence by Boratav and Pelz. ${ }^{11}$ On the other hand, the recent experiments by Camussi et al. ${ }^{9}$ and Kahalerras et al. ${ }^{13}$ did not give significant deviations between $\delta \zeta_{p}^{L}$ and $\delta \zeta_{p}^{T}$ and experiments by Noullez et $a l^{4}$ found $\delta \zeta_{p}^{T}$ comparable to the $\delta \zeta_{p}^{L}$ found in other experiments. For a simple quantification of the intermittency corrections it is common to give $\delta \zeta_{6}$. We do so in table I for the addressed experiments and simulations.

We would like to caution the reader of a too simplistic interpretation of table I. The detailed definitions of the $\delta \zeta_{p}^{L, T}$ slightly differ from experiment to experiment. In all low Reynolds number experiments, ${ }^{4,9-11}$ the scaling exponents could only be determined by employing the extended self similarity (ESS) method introduced by Benzi et al. ${ }^{14}$ In this method the structure functions $D_{p}(r)$ are plotted against $D_{3}^{*}(r)$, where $D_{3}^{*}(r)$ is the third order structure function defined with the modulus of the velocity difference and is experimentally found to scale with roughly the same expo- 
TABLE I. Intermittency corrections $\delta \xi_{6}^{L}$ and $\delta \xi_{6}^{T}$ (see the text for the definition) for the longitudinal and transversal sixth order structure functions for various numerical and experimental flows.

\begin{tabular}{cccccc}
\hline \hline Reference & Flow & $R e_{\lambda}$ & $-\delta \xi_{6}^{L}$ & $-\delta \xi_{6}^{T}$ & Remark \\
\hline van de Water et al. $^{8}$ & Shear flow & up to 600 & $0.18-0.20$ & $0.27-0.31$ & Normalized \\
Camussi and Benzi $^{10}$ & Jet flow & 250 & 0.25 & 0.38 & ESS \\
${\text { Camussi } \text { et } \text { al. }^{9}}$ & Wind tunnel flow & 37 & $0.19 \pm 0.03$ & $0.22 \pm 0.03$ & ESS \\
Boratav and Pelz $^{11}$ & Decaying numerical flow & $\sim 100$ & 0.23 & 0.43 & ESS \\
Noullez et al. & Jet flow & up to 600 & - & $0.25 \pm 0.10$ & ESS \\
This work & Forced numerical flow & 110 & $0.21 \pm 0.01$ & $0.43 \pm 0.01$ & ESS \\
This work & Forced numerical flow & 70 & $0.22 \pm 0.01$ & $0.43 \pm 0.01$ & ESS \\
\hline \hline
\end{tabular}

nent as $D_{3}(r)$, which includes the sign of $v(r) .{ }^{15}$ The ISR scaling exponent of such a plot is henceforth denoted as $\xi_{p}=\zeta_{p} / \zeta_{3}^{*}$. As in the ISR $\zeta_{3}^{L}=1$ according to the structure equation (3), one expects $\xi_{p}^{L}=\zeta_{p}^{L}$, if $\zeta_{3}^{* L}=\zeta_{3}^{L}$. In experiments, however, there are always small deviations from $\zeta_{3}^{* L}=1$; therefore, in principle $\zeta_{p}^{L}$ and $\xi_{p}^{L}$ could slightly differ; see also Ref. 16. For the transversal structure function $D_{3}^{* T}(r)$ there is no known relation as equation (3). Indeed, van de Water's group finds $\zeta_{3}^{* T}=1.08^{8}$ and they give normalized scaling exponents $\zeta_{p} / \zeta_{3}$ which we also call $\xi_{p}$. To date, there is no strict theoretical argument why ESS works so well. Note that for the present numerical simulation it does not work for odd order structure functions, taken without the modulus. ${ }^{16}$

What is the origin of the differences between the results reported in table I? One may think that it is the different geometry of the flows which causes the differences, in particular, a different strength of the local shear and of the anisotropy in the flow. Indeed, the shear in the flow of Ref. 8 is considerable and it is known that shear destroys ESS. ${ }^{17,18}$ On the other hand, at least $\delta \xi_{6}^{L}$ was found to be remarkably independent of different flow geometries. ${ }^{19}$ Also, the numerical flow of Ref. 11 which clearly shows $\delta \xi_{6}^{L} \neq \delta \xi_{6}^{T}$ is highly isotropic. But it is decaying which in Ref. 20 is speculated to be a possible origin of the observed ${ }^{11}$ discrepancy between longitudinal and transversal intermittency corrections.

In this paper we set out to determine the scaling exponents of the longitudinal and transversal structure functions for forced, statistically stationary numerical Navier-Stokes turbulence up to $R e_{\lambda}=110$. Our motivation is to contribute to clarifying the contradictionary picture reflected in table I. It is of general interest for the understanding of intermittency whether in Navier-Stokes dynamics not only the velocity field and derivatives thereof are independently scaling fields as analyzed in Ref. 21, but that there are already two independently scaling velocity fields $v^{L}(r)$ and $v^{T}(r)$. Indeed, we will find significant differences for the longitudinal and transversal scaling corrections, namely, to put the result in a nutshell, $\delta \xi_{6}^{L}=0.21 \pm 0.01$ and $\delta \xi_{6}^{T}=0.43 \pm 0.01$ in very good agreement with Boratav's result for decaying turbulence. $^{11}$

The question which immediately comes up is whether this finding originates in the anisotropy of the flow. Therefore, we carefully analyze the degree of anisotropy of the numerical flow. We find only limited anisotropy and only on the very large scale and therefore consider the different scal- ing of longitudinal and transversal structure functions the more remarkable.

The second point we examine is whether these different intermittency corrections $\delta \xi_{p}^{L}$ and $\delta \xi_{p}^{T}$ correspond to different hierarchies of the moments. Such hierarchies were suggested by She and Leveque ${ }^{12}$ for the $r$-averaged energy dissipation rate $\epsilon_{r}$, namely

$$
\frac{\left\langle\epsilon_{r}^{p+1}\right\rangle}{\left\langle\epsilon_{r}^{p}\right\rangle}=B_{p}^{\prime}\left(\frac{\left\langle\epsilon_{r}^{p}\right\rangle}{\left\langle\epsilon_{r}^{p-1}\right\rangle}\right)^{\beta^{3}}\left(\epsilon_{r}^{(\infty)}\right)^{1-\beta^{3}},
$$

$B_{p}^{\prime}$ constant, $\epsilon_{r}^{(\infty)}=\lim _{p \rightarrow \infty}\left(\left\langle\epsilon_{r}^{p+1}\right\rangle /\left\langle\epsilon_{r}^{p}\right\rangle\right)$; the SL parameter $\beta$ is therefore called the hierarchy parameter. Such a hierarchy means that the corresponding probability distribution function obeys a log-Poisson statistics. ${ }^{22}$

Ruiz Chavarria et al. $^{23}$ extended the idea of hierarchies to structure functions. Assuming Kolmogorov's refined similarity hypothesis $D_{p}(r) \sim\left\langle\epsilon_{r}^{p / 3}\right\rangle r^{p / 3}$ (Refs. 2,24) the structure function hierarchy can be derived from the SL hierarchy $(6)^{23,18}$ and reads as

$$
\frac{D_{p+1}(r)}{D_{p}(r)}=B_{p}^{\prime \prime}\left(\frac{D_{p}(r)}{D_{p-1}(r)}\right)^{\beta}\left(D_{(\infty)}\right)^{1-\beta},
$$

$B_{p}^{\prime \prime}$ constant, $D_{(\infty)}(r)=\lim _{p \rightarrow \infty}\left(D_{p+1}(r) / D_{p}(r)\right)=\left(r \epsilon_{r}^{(\infty)}\right)^{1 / 3}$.

We will calculate the hierarchy parameter $\beta$ both for the longitudinal and transversal structure functions, very carefully considering the systematic and statistical errors. First, we find significant deviations between $\beta^{L}$ and $\beta^{T}$. Second, we find a slight but also significant dependence of the hierarchy parameters $\beta^{L}$ and $\beta^{T}$ on the order of the moment which is not expected within the SL model.

The paper is organized as follows: In section II, we define the numerical flow and carefully check its isotropy, in section III we report on various scaling relations, employing ESS and the generalized ESS (GESS ${ }^{15,18}$ ); we also calculate the hierarchy parameters $\beta^{L}$ and $\beta^{T}$. In section IV we determine $\delta \xi_{p}^{L}$ and $\delta \xi_{p}^{T}$ within a reduced wave vector set approximation of the Navier-Stokes dynamics ${ }^{25-27}$ in which very large $R e_{\lambda}$ can be achieved. Conclusions are drawn in section V.

\section{SET UP OF THE FLOW AND CHECK OF ITS ISOTROPY}

The 3D incompressible Navier-Stokes equations are numerically solved on a $N^{3}$ grid with periodic boundary condi- 


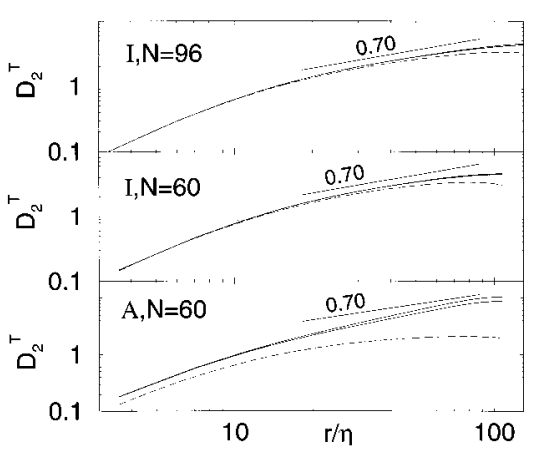

FIG. 1. Second order transversal structure functions $D_{2}^{T}(r)$ for the isotropic $N=96, \nu=0.006$ simulation $\left(R e_{\lambda}=110\right.$, upper), the isotropic $N=60$, $\nu=0.009$ simulation $\left(R e_{\lambda}=70\right.$, middle), and the anisotropic $N=60$, $\nu=0.009$ simulation $\left(R e_{\lambda}=70\right.$, bottom). The solid lines are calculated from the definition (2) of $D_{2}^{T}(r)$ (for the two directions being perpendicular to $r$ ); the dashed line is calculated from relation (4) which holds for perfect isotropy and homogeneity. For the anisotropic case anisotropy can be seen on all scales; also the ISR slope deviates from the expected value $\zeta_{2}=0.70$.

tions. Spherical truncation is used to reduce aliasing. For the isotropic flow simulation (denoted by " $I$ ") we force the system on the largest scale [wavevectors $k=(0,0, \pm 1) / L$, $\boldsymbol{k}=(0, \pm 1, \pm 1) / L, \quad \boldsymbol{k}=( \pm 1, \pm 1, \pm 1) / L$, and permutations thereof] with a forcing term as, e.g., described in Ref. 27. Units are fixed by picking the length scale $L=1$ and the average energy input rate ( $=$ the energy dissipation rate) $\epsilon=1$. The Taylor-Reynolds number is defined as $\operatorname{Re}_{\lambda}=u_{1, r m s} \lambda / \nu$, where $\lambda=u_{1, r m s} /\left(\partial_{1} u_{1}\right)_{r m s}$ is the Taylor length and $\nu$ the viscosity. Most of our results refer to $N=96$ and $\nu=0.006$, corresponding to a resolution of scales $r \geqslant 2 \pi L / N \approx 3 \eta$ and $R e_{\lambda}=110$. Time integrations of about 140 large eddy turnover times are performed. Averages are taken over space and time. To check the Reynolds number dependence we also did an isotropic $N=60, \nu=0.009$ simulation (240 large eddy turnovers) which has $R e_{\lambda}=70$. For a less isotropic flow simulation (denoted by " $A$ ") we only force one mode $k=(0,0,1) / L$. This simulation is done for $N=60, \nu=0.009$, for about 210 large eddy turnovers; it has $R e_{\lambda}=70$, too.

We checked the isotropy of the flow in several ways. - We calculated the structure functions for different space directions and compared them among each other. For the simulation "I" good agreement is found, for " $A$ " one space direction is distinguished as expected from the type of forcing, see figure 1 . Moreover, for the isotropic simulation we find less than $5 \%$ deviations between $\left\langle u_{1}^{2}\right\rangle,\left\langle u_{2}^{2}\right\rangle$, and $\left\langle u_{3}^{2}\right\rangle$. Note that $D_{2}^{L}(r=\pi)$ and $D_{2}^{T}(r=\pi)$ (we used $r=\pi$ as the

TABLE II. Energy input and dissipation rates for the three numerical simulations. The good agreement between the energy input and the total energy dissipation rate $\epsilon$ means statistical stationarity. The degree of agreement between the last two columns with 1 characterizes the degree of isotropy in the VSR.

\begin{tabular}{lcccc}
\hline \hline & Energy input & $\epsilon$ & $15 \nu\left\langle\left(\partial_{1} u_{1}\right)^{2}\right\rangle$ & $(15 / 2) \nu\left\langle\left(\partial_{2} u_{1}\right)^{2}\right\rangle$ \\
\hline $\mathrm{I}, N=96$ & 1 & 1.003 & 0.984 & 1.021 \\
$\mathrm{I}, N=60$ & 1 & 1.003 & 0.984 & 0.994 \\
$\mathrm{~A}, N=60$ & 1 & 1.004 & 0.931 & 0.851 \\
\hline \hline
\end{tabular}



FIG. 2. Third order structure function $D_{3}^{L}(r)$, directly calculated from the numerics (long dashed) and from Kolmogorov's structure equation (3) (solid). Also shown are $D_{3}^{* L}(r)$ (dashed-dotted) and $\left|D_{3}^{T}(r)\right|$ (short dashed). The data are for the isotropic $N=96$ simulation $\left(R e_{\lambda}=110\right)$.

largest possible space separation in numerical flow with periodic boundary conditions) do not equal $2\left\langle u_{i}^{2}\right\rangle(i=1,2$, or 3 ) as expected for experimental, isotropic flow at $r=\infty$. We find deviations up to $25 \%$ which means that the velocities are still correlated at the space distance of $r=\pi$. For the longitudinal velocities we find a positive correlation of about $25 \%$, for the transversal velocities we find a negative correlation of about $15 \%$. Geometrically, this means that there is a large scale eddy with diameter $\sim \pi$. We can not fully exclude that the results on scaling exponents we will report on are influenced by the flow geometry (periodic boundary conditions). They might be different for different geometries (e.g., those in experimental flows).

- We checked relation (4) which only holds for isotropy. ${ }^{1}$ For " $I$ " there are only large scale deviations, for " $A$ " deviations show up down to small scales; see figure 1 .

- We checked the relation (3), see figure 2. It holds for isotropic flow. The agreement is reasonable. However, there still is no developed inertial subrange due to the low $R e_{\lambda}$. The curve looks very similar to the experimental curve for comparable $R e_{\lambda}$; cf. Fig. 2 of Ref. 8. In particular, also the experimental curves bend down for large $r$. The reason for this of course is that at large scales the fluctuations are Gaussian and odd order moments vanish. We ascribe the deviations in the viscous subrange (VSR) to the lack of perfect convergence of odd moments. This difference remained even for as long averaging times as 140 large eddy turnovers. Also the relation $D_{3}^{T}(r)=0$ is not yet fulfilled for this low $R e_{\lambda}$, though the modulus of $D_{3}^{T}(r)$ is more than one decade smaller than the modulus of $D_{3}^{L}(r)$ for all scales, see figure 2.

- For perfect isotropy, the mean energy dissipation rate $\epsilon$ can be calculated from any component of the strain tensor $\partial_{i} u_{j}$, e.g.,

$$
\epsilon=15 \nu\left\langle\left(\partial_{1} u_{1}\right)^{2}\right\rangle=\frac{15}{2} \nu\left\langle\left(\partial_{2} u_{1}\right)^{2}\right\rangle .
$$

For the isotropic flow, these relations hold very well see table II for the anisotropic one there are deviations up to $15 \%$.

- For an isotropic flow, the isotropy coefficient, ${ }^{28}$ 


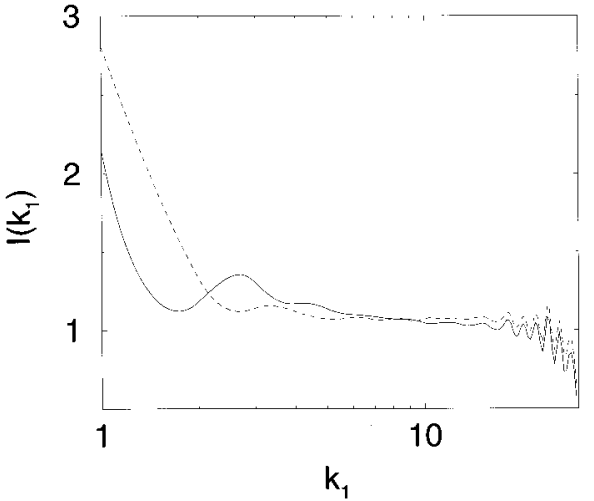

FIG. 3. Isotropy coefficient $I\left(k_{1}\right)$, eq. (9), for the simulations "I'" (solid) and "A" (dashed) $(N=60)$.

$$
I\left(k_{1}\right)=\frac{E_{11}\left(k_{1}\right)-k_{1} \partial E_{11}\left(k_{1}\right) / \partial k_{1}}{2 E_{22}\left(k_{1}\right)}
$$

which compares the longitudinal and transversal energy spectra $E_{11}\left(k_{1}\right)$ and $E_{22}\left(k_{1}\right)$ should become $1 .{ }^{29} I\left(k_{1}\right)$ is shown in figure 3. Indeed, in general $I\left(k_{1}\right)$ is closer to 1 for "I" than it is for "A." We do not quite understand the bump around $k_{1}=2.5$ in $I\left(k_{1}\right)$ in the isotropic simulation. We tend to ascribe it to the forcing of the modes $( \pm 1, \pm 1, \pm 1) / L$. The wiggles for very large $k_{1}$ are numerical artifacts because of the derivative in eq. (9).

\section{SCALING EXPONENTS FOR LONGITUDINAL AND TRANSVERSAL STRUCTURE FUNCTIONS}

To determine the degree of intermittency in the longitudinal and transversal structure functions we employ a type of $\mathrm{ESS}^{14,30,18}$ by calculating generalized structure functions,

$$
G_{p}(r)=\frac{D_{p}(r)}{\left(D_{3}^{*}(r)\right)^{p / 3}}
$$

and plotting them vs $D_{3}^{*}$ ("compensated ESS plot;", ",31); see figure 4 . The intermittency exponents $\delta \xi_{6}$ (the ISR slopes in figure 4) for the longitudinal and transversal structure functions are clearly different; the transversal signal shows considerably more intermittency. No dependence on $R e_{\lambda}$ is found. The values of $\delta \xi_{6}^{L, T}$ for the isotropic $R e_{\lambda}=110$ and $R e_{\lambda}=70$ simulations are given in table I. Surprisingly, also the anisotropic simulation " $A$ " approximately has the same scaling exponents, namely $\delta \xi_{6}^{L}=-0.23 \pm 0.01$ and $\delta \xi_{6}^{T}=-0.40 \pm 0.01$; see figure 4 . Therefore, in what follows we will only focus on the isotropic simulation "I." Our results for various $\delta \xi_{p}$ determined as in figure 4 are summarized in figure 5 .

It can be seen that the intermittency corrections $\delta \xi_{p}^{L}$ and $\delta \xi_{p}^{T}$ clearly deviate throughout, i.e., transversal velocity fluctuation are much more intermittent than longitudinal ones. Though it has been known for many years that the transversal velocity gradient $\partial_{2} u_{1}$ is more intermittent than the longitudinal one $\partial_{1} u_{1}$, see, e.g., Ref. 32-in our simulations we have flatnesses of $F_{\partial_{1} u_{1}}=4.9, \quad F_{\partial_{2} u_{1}}=7.0, \quad F_{\partial_{1} u_{2}-\partial_{2} u_{1}}$ $=7.0-$ it is not trivial that this difference, probing the VSR,
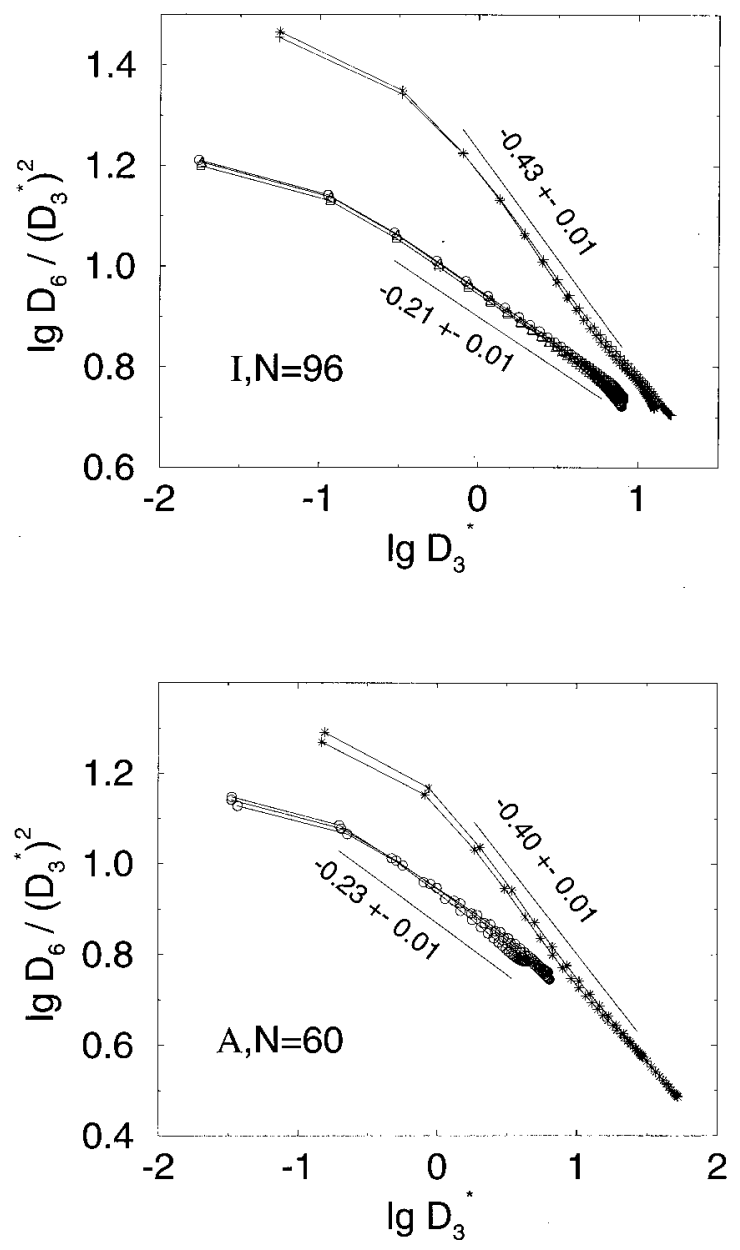

FIG. 4. Compensated ESS type plots for $D_{6}^{L} /\left(D_{3}^{* L}\right)^{2}$ vs $D_{3}^{* L}$ (circles) and $D_{6}^{T} /\left(D_{3}^{* T}\right)^{2}$ vs $D_{3}^{* T}$ (stars). The ISR slopes are, respectively, $\delta \xi_{6}^{L}$ and $\delta \xi_{6}^{T}$. The upper part of the figure refers to the isotropic simulation with $N=96$, the lower one to the anisotropic one with $N=60$.

is carried on into the ISR. In Ref. 11 the different degrees of intermittency were associated with different types of structures: Longitudinal fluctuations with strain like structures, transversal fluctuations with vorticity like structures-which both is in keeping with the definitions of strain and vorticity, respectively.

Let us discuss our results on $\delta \xi_{p}^{L, T}$ in figure 5 in more detail. The values for $\delta \xi_{p}^{L}$ are well described by the SL model fit eq. (5) with the SL values $C_{0}=2$, $\beta=(2 / 3)^{1 / 3} \approx 0.874$ as found for many other isotropic, even low $R e_{\lambda}$ number, experimental or numerical flows. ${ }^{2,14,15}$ For the physical interpretation of the parameters in the SL model we refer to Refs. 12 and 22. From a phenomenological point of view, one could consider eq. (5) simply as a two parameter fit of the $\xi_{p}$ 's. The two SL parameters for the transversal scaling exponents can be viewed as a simple way to quantify the degree of intermittency.

We now suggest a method to replace this one twoparameter-fit by two one-parameter fits. To do so, we employ generalized extended self similarity $\left(\right.$ GESS $\left.^{15,18}\right)$ and plot $G_{p}$ 


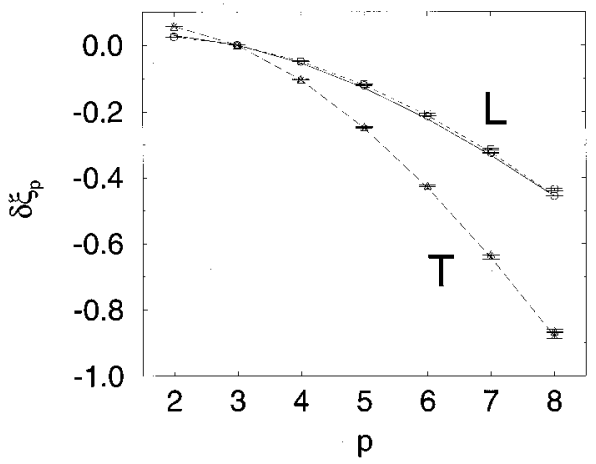

FIG. 5. Intermittency corrections $\delta \xi_{p}^{L}$ (circles for $N=96$, squares $N=60$ ) and $\delta \xi_{p}^{T}$ (crosses $N=96$, triangles $N=60$ ) from the isotropic numerical simulations. The dashed lines are 1-parameter fits within the SL model where the $\beta$ 's have been taken fixed from the averaged fits of the GESS type plot, i.e., $\beta^{L}=0.947$ and $\beta^{T}=0.870$. The one free fit parameter is thus $C_{0}$. We obtain the shown remarkably good fits with $C_{0}^{L}=9.3$ for the longitudinal data (short dashed) and $C_{0}^{T}=3.7$ for the transversal data (long dashed). The standard SL fit ${ }^{12} \beta=(2 / 3)^{1 / 3}, C_{0}=2$ fits the longitudinal corrections also pretty well, see the solid line.

vs $G_{q}$, see figure 6 . The slope $\rho_{p, q}$ of such a plot is by definition (10),

$$
\rho_{p, q}=\frac{\xi_{p}-p / 3}{\xi_{q}-q / 3} .
$$

For fixed $p, q, \rho_{p, q}^{L}$, and $\rho_{p, q}^{T}$ are significantly different, see table III. We checked this result very carefully. The small error bars in $\rho_{p, q}$ result from linear regressions in GESS plots as in figure 6 and in addition from an averaging over the different space directions. We also checked this for smaller $R e_{\lambda}=70$; the deviations in comparison to the results given in table III are never larger than $0.5 \%$. Moreover, to

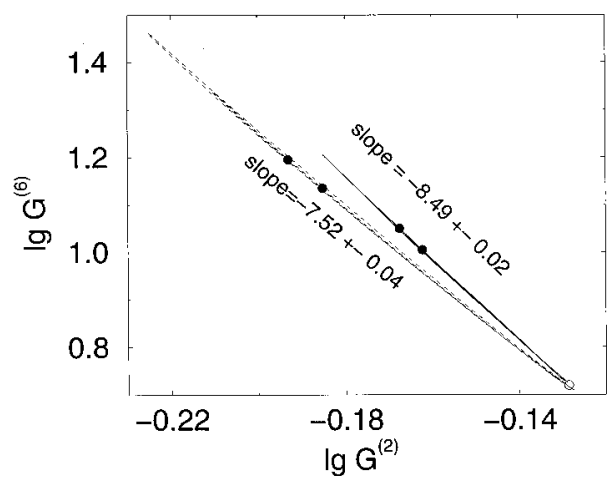

FIG. 6. GESS type plot $G^{(6)}(r)$ vs $G^{(2)}(r)$ for both the longitudinal (solid) and the transversal (dashed) $G$-structure functions for the isotropic simulations. The slopes of these curves are $\rho_{6,2}^{L}=-8.49 \pm 0.02$ and $\rho_{6,2}^{T}=-7.52 \pm 0.04$, respectively. The errors result from a linear regression of every single curve, from weighted averaging of the results for different space directions, and different $R e_{\lambda}$. The hardly distinguishable lines within the two bunches of curves are the results for different directions and different Reynolds numbers $R e_{\lambda}=110$ and $R e_{\lambda}=70$. The good agreement within the bunches means good isotropy and independence of the scaling exponents from $R e_{\lambda}$. Note that in this type of plot the far VSR collapses into the upper left point of these curves. The filled bullets refer to $r=10 \eta$ for $R e_{\lambda}=110$ (left one) and $R e_{\lambda}=70$ (right one). The open bullet refers to the outer length scale $r=L$.
TABLE III. $\rho_{p, q}^{L, T}$ for various pairs $p, q$ from GESS type plots as in figure 6 . By definition, $\rho_{p, q}=\rho_{q, p}^{-1}$. The errors are purely statistical ones. In the third and fifth column, we give the $\beta$ 's resulting from eq. (12). Note that GESS implies $\rho_{p, s}=\rho_{p, q} \rho_{q, s}$. This relation can be used to check the quality of GESS.

\begin{tabular}{ccccc}
\hline \hline$p, q$ & $\rho_{p, q}^{L}$ & $\beta^{L}$ & $\rho_{p, q}^{T}$ & $\beta^{T}$ \\
\hline 2,4 & $-0.5093 \pm 0.0004$ & $0.973 \pm 0.001$ & $-0.5440 \pm 0.0011$ & $0.877 \pm 0.003$ \\
2,5 & $-0.2073 \pm 0.0002$ & $0.964 \pm 0.001$ & $-0.2272 \pm 0.0006$ & $0.875 \pm 0.002$ \\
2,6 & $-0.1176 \pm 0.0002$ & $0.957 \pm 0.001$ & $-0.1318 \pm 0.0004$ & $0.873 \pm 0.002$ \\
2,7 & $-0.0774 \pm 0.0001$ & $0.952 \pm 0.001$ & $-0.0884 \pm 0.0003$ & $0.872 \pm 0.002$ \\
2,8 & $-0.0555 \pm 0.0001$ & $0.947 \pm 0.001$ & $-0.0645 \pm 0.0003$ & $0.871 \pm 0.003$ \\
4,5 & $0.4072 \pm 0.0001$ & $0.947 \pm 0.001$ & $0.4177 \pm 0.0003$ & $0.870 \pm 0.002$ \\
4,6 & $0.2311 \pm 0.0001$ & $0.941 \pm 0.001$ & $0.2423 \pm 0.0004$ & $0.869 \pm 0.003$ \\
4,7 & $0.1521 \pm 0.0002$ & $0.937 \pm 0.001$ & $0.1625 \pm 0.0005$ & $0.868 \pm 0.003$ \\
4,8 & $0.1092 \pm 0.0003$ & $0.933 \pm 0.002$ & $0.1186 \pm 0.0005$ & $0.867 \pm 0.003$ \\
5,6 & $0.5675 \pm 0.0002$ & $0.935 \pm 0.001$ & $0.5800 \pm 0.0005$ & $0.867 \pm 0.003$ \\
5,7 & $0.3736 \pm 0.0004$ & $0.931 \pm 0.002$ & $0.3890 \pm 0.0008$ & $0.867 \pm 0.003$ \\
5,8 & $0.2682 \pm 0.0006$ & $0.928 \pm 0.002$ & $0.2840 \pm 0.0010$ & $0.866 \pm 0.004$ \\
6,7 & $0.6583 \pm 0.0005$ & $0.927 \pm 0.002$ & $0.6708 \pm 0.0008$ & $0.865 \pm 0.004$ \\
6,8 & $0.4725 \pm 0.0008$ & $0.924 \pm 0.003$ & $0.4901 \pm 0.0014$ & $0.863 \pm 0.005$ \\
7,8 & $0.7177 \pm 0.0007$ & $0.922 \pm 0.003$ & $0.7308 \pm 0.0010$ & $0.860 \pm 0.005$ \\
\hline \hline
\end{tabular}

make sure that our numbers are well converged, we also averaged over only 30,60, and 90 large eddy turnovers rather than 140; still, the result is the same; the deviations are smaller than the error bars.

To quantify the quality to which GESS holds we checked the relation $\rho_{p, s}=\rho_{p, q} \rho_{q, s}$, implied by GESS, for various $p, q$. Table III allows the reader to do so. Neither for the $\rho_{p, q}^{L}$ nor for the $\rho_{p, q}^{T}$ did we find a single example where there were deviations larger than the error bars. For example, $\rho_{2,4}^{L} \cdot \rho_{4,6}^{L}=0.1177 \pm 0.0005$ which equals $\rho_{2,6}^{L}$ within the error bars.

The error bars up to now stem from statistics. One would like to be able to judge the size of the systematic errors. Therefore, in figure 7 we display the local slope of the curves in figure 6 . Both the longitudinal and the transversal local slope slightly increase (modulus-wise) with increasing scale (i.e., from left to right), which shows the limitations of the above statement that GESS is fulfilled with remarkable



FIG. 7. Local slopes of figure 6 for the $R e_{\lambda}=110$ simulation. The two dashed lines are for the two different space directions for the transversal structure functions, the three solid lines are for the three space directions of the longitudinal structure functions. If we calculate the average (for scales up to $r=2.0 \approx 100 \eta$ ) we obtain $\rho_{6,2}^{L}=-8.36 \pm 0.14$ and $\rho_{6,2}^{T}=-7.36 \pm 0.26$. The arrows refer to $10 \eta$ and $L$, respectively. 


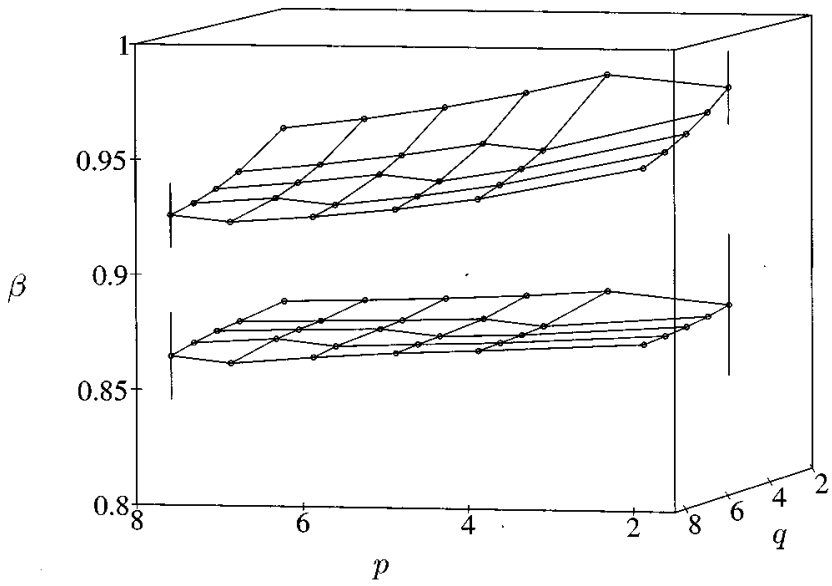

FIG. 8. The longitudinal and transversal hierarchy parameters $\beta_{p, q}^{L}$ (upper) and $\beta_{p, q}^{T}$ (lower), respectively. The data are taken from table III, i.e., for the "I" simulation with $R e_{\lambda}=110$, the averaging time is 140 large eddy turnovers. To get an idea of the size of the error, two error bars are drawn, representing the much larger systematic errors rather than the statistical ones. First, we observe that $\beta^{L}$ and $\beta^{T}$ are clearly different. Second, a slight dependence of $\beta^{L, T}$ on $p, q$ is seen.

quality. The error bar calculated from averaging the local slope is much bigger than above statistical error. From averaging up to the scale $r=2.0 \approx 100 \eta$ we obtain $\rho_{6,2}^{L}=-8.36 \pm 0.14$ and $\rho_{6,2}^{T}=-7.36 \pm 0.26$. The numbers for the $\rho_{p, q}^{L, T}$ from table III are within the (now about ten times larger) error bars. These systematic errors are one order of magnitude bigger than the purely statistical ones in table III. We summarize the values of $\rho_{p, q}^{L, T}$ (and their error bars) determined in this way in table IV. Note, however, that the deviations between $\rho_{p, q}^{L}$ and $\rho_{p, q}^{T}$ and correspondingly also between the resulting $\beta$ 's (see below) are still statistically significant.

Within the SL model, the $\rho_{p, q}$ 's only depend on $\beta$, not on any other parameter,

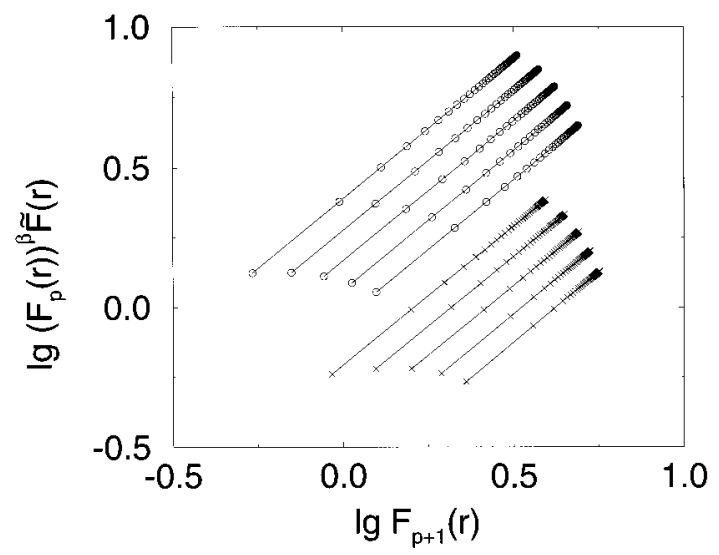

FIG. 9. The $\log -\log$ plot of $F_{p+1}(r)$ vs $\left(F_{p}(r)\right)^{\beta} \widetilde{F}(r)$ for various $p$ for the longitudinal and transversal structure functions, $\beta^{L}=0.947, \beta^{T}=0.870$. The lines are arbitrarily shifted in order for the slopes to be visible. The upper 5 lines are for the longitudinal structure functions, $p=3$ (upper) to $p=7$ (lower), the lower 5 lines are for the transversal structure functions, $p=3$ (upper) to $p=7$ (lower).
TABLE IV. $\rho_{p, q}^{L, T}$ for various pairs $p, q$ determined from the local slope of the GESS type plots, cf. figure 7. The errors are the systematic ones, stemming from the local slope not being constant. In the third and fifth columns, we again give the $\beta$ 's and their errors resulting from eq. (12). Averaging the $\beta$ 's determined this way gives $\beta^{L}=0.930$ and $\beta^{T}=0.855$.

\begin{tabular}{ccccc}
\hline \hline$p, q$ & $\rho_{p, q}^{L}$ & $\beta^{L}$ & $\rho_{p, q}^{T}$ & $\beta^{T}$ \\
\hline 2,4 & $-0.514 \pm 0.005$ & $0.958 \pm 0.016$ & $-0.556 \pm 0.012$ & $0.846 \pm 0.031$ \\
2,5 & $-0.210 \pm 0.003$ & $0.950 \pm 0.014$ & $-0.234 \pm 0.007$ & $0.848 \pm 0.027$ \\
2,6 & $-0.120 \pm 0.002$ & $0.944 \pm 0.012$ & $-0.136 \pm 0.005$ & $0.849 \pm 0.025$ \\
2,7 & $-0.079 \pm 0.002$ & $0.940 \pm 0.012$ & $-0.092 \pm 0.003$ & $0.851 \pm 0.023$ \\
2,8 & $-0.057 \pm 0.001$ & $0.936 \pm 0.011$ & $-0.067 \pm 0.003$ & $0.852 \pm 0.021$ \\
4,5 & $0.409 \pm 0.001$ & $0.936 \pm 0.011$ & $0.420 \pm 0.003$ & $0.851 \pm 0.021$ \\
4,6 & $0.233 \pm 0.002$ & $0.931 \pm 0.010$ & $0.245 \pm 0.003$ & $0.853 \pm 0.020$ \\
4,7 & $0.153 \pm 0.002$ & $0.927 \pm 0.010$ & $0.165 \pm 0.003$ & $0.855 \pm 0.018$ \\
4,8 & $0.110 \pm 0.001$ & $0.924 \pm 0.011$ & $0.120 \pm 0.003$ & $0.856 \pm 0.018$ \\
5,6 & $0.569 \pm 0.002$ & $0.926 \pm 0.010$ & $0.582 \pm 0.003$ & $0.855 \pm 0.018$ \\
5,7 & $0.376 \pm 0.002$ & $0.923 \pm 0.010$ & $0.391 \pm 0.004$ & $0.857 \pm 0.018$ \\
5,8 & $0.270 \pm 0.003$ & $0.920 \pm 0.011$ & $0.286 \pm 0.004$ & $0.858 \pm 0.018$ \\
6,7 & $0.660 \pm 0.002$ & $0.919 \pm 0.011$ & $0.672 \pm 0.003$ & $0.858 \pm 0.018$ \\
6,8 & $0.475 \pm 0.004$ & $0.917 \pm 0.012$ & $0.491 \pm 0.005$ & $0.859 \pm 0.018$ \\
7,8 & $0.719 \pm 0.003$ & $0.914 \pm 0.014$ & $0.731 \pm 0.004$ & $0.860 \pm 0.019$ \\
\hline \hline
\end{tabular}

$$
\rho_{p, q}^{S L}=\frac{\left(1-\beta^{p}\right)-(p / 3)\left(1-\beta^{3}\right)}{\left(1-\beta^{q}\right)-(q / 3)\left(1-\beta^{3}\right)} .
$$

For each $\rho_{p, q}$ we calculate $\beta_{p, q}$, resulting from equation (12), and its error; see table III and table IV. If the SL model were exact, $\beta$ should not depend on $p$ and $q$.

In figure 8 we offer a 3D plot of $\beta_{p, q}^{L, T}$, together with the error bars resulting from the (larger) systematic errors of $\rho$; cf. table IV. From figure 8 the difference between $\beta^{L}$ and $\beta^{T}$ seems to be significant. This result is at variance with the result of Camussi and Benzi ${ }^{10}$ who obtained that the difference of both $\beta^{L}$ and $\beta^{T}$ to the SL value $\beta=(2 / 3)^{1 / 3} \approx 0.874$ is at most $1.2 \%=0.010$.

Another feature of figure 8 is that $\beta_{p, q}^{L}$ shows a small trend towards smaller values for larger $p, q$ which is not expected within the SL model. If we average over all $\beta_{p, q}$ nevertheless, we obtain $\beta^{L}=0.947$ and $\beta^{T}=0.870$.

Knowing $\beta$, there is only the parameter $C_{0}$ left in eq. (5). If we take the above mean values $\beta^{L}=0.947$ and $\beta^{T}=0.870$, we obtain as best fits to the $\xi_{p}$ data in figure 5, $C_{0}^{L}=9.3$ (the $\chi^{2}$ of the fit is $\chi^{2}=10$ ) and $C_{0}^{T}=3.7$ (with $\chi^{2}=1$ ), excellently describing the numerical data. We do not ascribe any physical meaning to the parameter values obtained in our fit. Note that for our $\xi^{L}$ data this fit is superior to the SL model with the original parameter values $\beta=(2 / 3)^{1 / 3}, \quad C_{0}=2$. If we choose the SL value $\beta^{L}=\beta^{T}=(2 / 3)^{1 / 3}$ we obtain $C_{0}^{L}=1.97$ with $\chi^{2}=10^{3}$ and $C_{0}^{T}=3.9$ with $\chi^{2}=0.8$. (The $\chi^{2}$ values for $C_{0}^{L}$ are larger than those for $C_{0}^{T}$ as the errors of the $\delta \xi_{p}^{L}$ are smaller than those of $\delta \xi_{p}^{T}$.)

We now directly check the hierarchies of the structure functions. ${ }^{12,18,23}$ From eq. (7) it is easy to derive ${ }^{18,23}$

$$
F_{p+1}(r)=B_{p}\left(F_{p}(r)\right)^{\beta} \widetilde{F}(r),
$$

with

$$
F_{p+1}=\frac{D_{p+1}(r)}{D_{p}(r)}
$$


TABLE V. Slopes of $\lg F_{p+1}(r)$ vs $\lg \left(\left(F_{p}(r)\right)^{\beta} \widetilde{F}(r)\right)$, varying $r$. According to eq. (13), the slopes should be 1 , which is pretty well fulfilled for $\beta^{L}=0.947$ and $\beta^{T}=0.870$. For comparision, we also give the slopes if the SL value $\beta^{L}=(2 / 3)^{1 / 3}$ is used for the longitudinal structure function. The deviations of the slope to 1 are larger. The constants $B_{p}$ in (13) show a slight $p$-dependence.

\begin{tabular}{ccccccc}
\hline \hline & $\beta^{L}=0.947$ & \multicolumn{3}{c}{$\beta^{T}=0.870$} & & $\beta^{L}=(2 / 3)^{1 / 3}$ \\
$p$ & Slope $L$ & $B_{p}^{L}$ & Slope $T$ & $B_{p}^{T}$ & Slope $L$ & $B_{p}^{L}$ \\
\hline 3 & $0.9984 \pm 0.0001$ & 0.975 & $0.9988 \pm 0.0002$ & 0.981 & $0.9988 \pm 0.0001$ & 0.981 \\
4 & $0.9995 \pm 0.0001$ & 0.944 & $0.9993 \pm 0.0004$ & 0.961 & $0.9936 \pm 0.0001$ & 0.969 \\
5 & $1.0020 \pm 0.0004$ & 0.925 & $1.0000 \pm 0.0005$ & 0.951 & $0.9895 \pm 0.0002$ & 0.967 \\
6 & $1.0050 \pm 0.0007$ & 0.910 & $1.0020 \pm 0.0007$ & 0.945 & $0.9854 \pm 0.0004$ & 0.970 \\
7 & $1.0080 \pm 0.0010$ & 0.899 & $1.0040 \pm 0.0016$ & 0.940 & $0.9810 \pm 0.0007$ & 0.977 \\
\hline \hline
\end{tabular}

and

$$
\widetilde{F}(r)=\left(\frac{D_{6}(r)}{\left(D_{3}^{*}(r)\right)^{1+\beta^{3}}}\right)^{(1-\beta) /\left[3\left(1-\beta^{3}\right)\right]} .
$$

With the mean $\beta$ 's obtained above, we plot $F_{p+1}(r)$ vs $\left(F_{p}(r)\right)^{\beta} \widetilde{F}(r)$. If eq. (13) and equivalently eq. (7) hold, the slope should be 1 . Indeed, the slope is very close to 1 , see figure 9 and table $\mathrm{V}$, which gives further support for $\beta^{L}$ and $\beta^{T}$ being different. The best agreement is found for $p=4$ to $p=6$; see table $\mathrm{V}$. The reason is that the mean $\beta$ 's best agree with $\beta_{p, q}$ if $p, q$ are around 4-6; see table III. For the other $p$ one could improve the fit by using the corresponding $\beta_{p, q}$; however, note that the sixth order structure function always enters via $\widetilde{F}(r)$, cf. eq. (15).

Moreover, we find a $p$ dependence of the prefactor $B_{p}$ in eq. (13). Therefore, determining $\beta$ from eq. (13) by plotting $\log F_{p+1}(r)$ vs $\log F_{p}(r)$ for fixed $r$ as a function of $p$ as done in Refs. 9 and 33 does not seem to be possible here.

\section{SCALING RELATIONS WITHIN REWA}

Very large $R e_{\lambda}$ in numerical turbulent flow can be achieved in the reduced wave vector set approximation (REWA) of the Navier-Stokes equation. ${ }^{25-27,34,35}$ REWA uses a reduced, geometrically scaling subset of wavevectors on which the Navier-Stokes equation is solved. Here we choose a basic set of 50 modes per level. Very high TaylorReynolds numbers up to $R e_{\lambda}=7 \times 10^{4}$ (Refs. 27,34) can be achieved, however, flow structures are underrepresented ${ }^{31}$ and the intermittency corrections are strongly underestimated. $^{27,35}$

We redid ESS types plots for REWA for $R e_{\lambda}=8 \times 10^{2}$ and for $R e_{\lambda}=1.4 \times 10^{5}$ for both the longitudinal and the transversal sixth order structure functions, see figure 10. There is no detectable difference between the longitudinal and transversal scaling exponents. The absolute value $\delta \xi_{6}^{L} \sim \delta \xi_{6}^{T} \sim-0.009$ is much smaller (modulo wise) than the experimental or above numerical value $\delta \xi_{6}^{L} \sim-0.21$, as extensively analyzed and discussed in the previous work on REWA. ${ }^{25-27,34,35}$ Note, however, that the relative error for the $\delta \xi$ 's is much larger than in the full numerical simulations - we cannot exclude different degrees of intermittency for $D_{p}^{L}$ and $D_{p}^{T}$ within REWA.

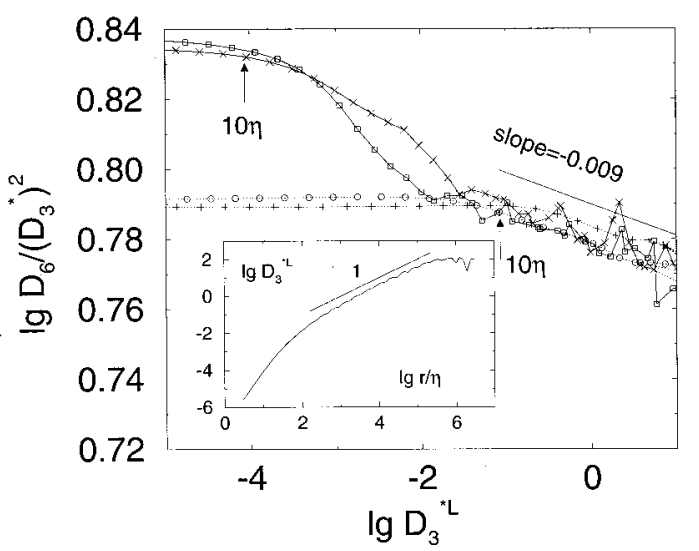

FIG. 10. Compensated ESS plot for the sixth order structure functions for the REWA calculation with 50 modes per level. The longitudinal and the transversal structure functions show the same slope $\delta \xi_{p}=-0.009$. Squares are for $R e_{\lambda}=1.4 \times 10^{5}$, longitudinal; crosses for $R e_{\lambda}=1.4 \times 10^{5}$, transversal; circles for $R e_{\lambda}=8 \times 10^{2}$, longitudinal; plusses for $R e_{\lambda}=8 \times 10^{2}$, transversal. The two arrows indicate the scale $10 \eta$ for the simulation with the higher (left arrow) and the lower (right arrow) Reynolds number, respectively. The inset shows the third order structure function $D_{3}^{* L}(r)$ (for $\left.\operatorname{Re}_{\lambda}=1.4 \times 10^{5}\right)$ in order to give an idea to what scale $r / \eta$ the data in the figure correspond.

We do not know whether our results on REWA indicate that the differences between the scaling of $D_{p}^{T}$ vs $D_{3}^{* T}$ and $D_{p}^{L}$ vs $D_{3}^{* L}$ observed in the above full numerical simulations for small $R e_{\lambda}$ are finite $R e_{\lambda}$ effects or whether they are artefacts of the REWA thinning of large wavevectors, ${ }^{25,27,31}$ connected to the suppression of small scale structures. Such structures are associated with the different scaling of longitudinal and transversal structure functions in Ref. 11. As within REWA no $R e_{\lambda}$ dependence of the $\delta \xi^{L}$ and $\delta \xi^{T}$ is observed (see figure 10); we favor the second interpretation.

We also tried GESS type scaling within REWA. No statistically significant deviations between the $\rho_{p, q}^{L}$ and the $\rho_{p, q}^{T}$ were found.

\section{SUMMARY, CONCLUSIONS, AND OUTLOOK}

To summarize, we offer strong evidence that the transversal velocity fluctuations show stronger intermittency than the longitudinal ones. Our numerical values for the longitudinal and the transversal scaling exponents $\xi_{p}^{L}$ and $\xi_{p}^{T}$ for forced stationary turbulence agree very well with those of Boratav and Pelz for decaying turbulence; ${ }^{11}$ see table I. This finding is independent of $R e_{\lambda}$, at least for the relatively low $R e_{\lambda}$ we examined. For an anisotropic flow we essentially obtained the same scaling exponents. Only for the REWA calculations which underrepresent the small scale structures of the flow we do not find a statistically significant deviation between $\delta \xi_{p}^{L}$ and $\delta \xi_{p}^{T}$, however, the relative error is much bigger than for the full simulations.

We reiterate that $\zeta_{2}^{L}=\zeta_{2}^{T}$ because of relation (4); a generalization of this equality to higher order moments $p>2$ is wrong.

GESS is fulfilled with satisfactory precision for both longitudinal and transversal structure functions. The GESS scaling exponents $\rho_{p, q}^{L}$ and $\rho_{p, q}^{T}$ are different. This result is the more remarkable, as those of the longitudinal velocity struc- 
ture function agree with those of an active ${ }^{36}$ or passive scalar ${ }^{18}$ or even with those calculated for the magnetic field in MHD; ${ }^{37}$ see table 1 of Ref. 18.

The She-Leveque hierarchy parameters $\beta^{L, T}$ following from the $\rho^{L, T}$ exponents consequently also differ. Both show a weak dependence on $p, q$ which is not expected within the She-Leveque model.

In the whole analysis we took great care of systematic and statistical errors to get significant statements.

To conclude, there seem to exist independently scaling velocity fields $v^{L}(r)$ and $v^{T}(r)$, i.e., the Navier-Stokes dynamics seems to make use of this degree of freedom being allowed by symmetry. It is very likely that the two different scaling velocities fields will also be reflected in the flow geometry.

A more complete discription of the statistics of the velocity field was recently suggested by L'vov, Podivilov, and Procaccia. ${ }^{38}$ These authors point out that because of rotational symmetry only the $\mathrm{SO}(3)$ irreducible amplitudes of the velocity structure tensors should obey clean scaling. In Ref. 39 we analyze the scaling properties of these amplitudes numerically and show (for fourth order moments) how they are connected to the longitudinal and transversal structure functions.

Presently, neither the longitudinal nor the transversal scaling exponents, nor the scaling exponents of the irreducible $\mathrm{SO}(3)$ invariants of the velocity correlations ${ }^{38}$ can be calculated analytically from the Navier-Stokes dynamics. Many phenomenological models based on various views on how intermittency develops are able to fit the longitudinal intermittency corrections. It should be possible to derive also the transversal intermittency corrections in the framework of the thinking these models are based on in order to check their consistency. The ultimate goal, however, must be to derive both longitudinal and transversal scaling exponents from the Navier-Stokes equation.

\section{ACKNOWLEDGMENTS}

We thank Luca Biferale and O. Boratav for careful reading of the manuscript and Rolf Nicodemus for drawing figure 8. Support for this work by the Deutsche Forschungsgemeinschaft (DFG) under Grant No. SFB185-D3 and by the German-Israel Foundation (GIF) is acknowledged. The HLRZ Jülich supplied us with computer time. D. L. acknowledges the hospitality of Leo Kadanoff and the University of Chicago, where part of the work was done, and support by MRSEC.

${ }^{1}$ A. S. Monin and A. M. Yaglom, Statistical Fluid Mechanics (The MIT Press, Cambridge, MA, 1975).

${ }^{2}$ U. Frisch, Turbulence (Cambridge University Press, Cambridge, 1995).

${ }^{3}$ G I. Taylor, "The spectrum of turbulence," Proc. R. Soc. London, Ser. A 164, 476 (1938).

${ }^{4}$ A. Noullez, G. Wallace, W. Lempert, R. B. Miles, and U. Frisch, "Transverse velocity increments in turbulent flow using the RELIEF technique," J. Fluid Mech. 339, 287 (1997).

${ }^{5}$ The relations between $D_{4}^{T}(r)$ and $D_{4}^{L}(r)$ given in M. Ould-Rouis, R. A. Antonia, Y. Zhu, and F. Anselmet, "Turbulent pressure structure function," Phys. Rev. Lett. 77, 2222 (1996), are restricted to the approximation of classical scaling exponents.

${ }^{6}$ S. G. Saddoughi and S. V. Veeravalli, "Local isotropy in turbulent bound- ary layers at high Reynolds numbers," J. Fluid Mech. 268, 333 (1994).

${ }^{7}$ J. Herweijter and W. van de Water, "Transverse structure functions of turbulence,' in Advance in Turbulence V, edited by R. Benzi (Kluwer Academic, New York, 1995), p. 210.

${ }^{8}$ W. van de Water and J. Herweijter, "High order structure functions of turbulence,"' preprint, Eindhoven, 1996.

${ }^{9}$ R. Camussi, D. Barbagallo, G. Guj, and F. Stella, "Transverse and longitudinal scaling laws in non-homogeneous low Re turbulence,' Phys. Fluids 8, 1181 (1996).

${ }^{10}$ R. Camussi and R. Benzi, "Hierarchy of transverse structure functions," Phys. Fluids 9, 257 (1997).

${ }^{11} \mathrm{O}$. N. Boratav and R. B. Pelz, "Structures and structure functions in the inertial range of turbulence," Phys. Fluids 9, 1400 (1997).

${ }^{12}$ Z. S. She and E. Leveque, "Universal scaling laws in fully developed turbulence,” Phys. Rev. Lett. 72, 336 (1994).

${ }^{13} \mathrm{H}$. Kahalerras, Y. Malecot, and Y. Gagne, "Transverse velocity structure functions in developed turbulence," in Advance in Turbulence VI, edited by S. Gavrilakis, L. Michiels, and P. A. Monkewitz (Kluwer Academic, New York, 1996), p. 235.

${ }^{14}$ R. Benzi, S. Ciliberto, R. Tripiccione, C. Baudet, F. Massaioli, and S. Succi, "Extended self-similarity in turbulent flows," Phys. Rev. E 48, R29 (1993).

${ }^{15}$ R. Benzi, L. Biferale, S. Ciliberto, M. V. Struglia, and R. Tripiccione, "On the intermittent energy transfer at viscous scales in turbulent flows," Europhys. Lett. 32, 709 (1995).

${ }^{16} \mathrm{~S}$. Grossmann, D. Lohse, and A. Reeh, "Application of extended self similarity in turbulence,' Phys. Rev. E 56, 5474 (1997).

${ }^{17}$ S. Stolovitzky and K. R. Sreenivasan, "Scaling of structure functions,", Phys. Rev. E 48, R33 (1993).

${ }^{18}$ R. Benzi, L. Biferale, S. Ciliberto, M. V. Struglia, and R. Tripiccione, “'Generalized scaling in fully developed turbulence,' Physica D 96, 162 (1996).

${ }^{19}$ A. Arneodo et al., "Structure functions in turbulence, in various flow configurations, at Reynolds number between 30 and 5000, using extended self-similarity," Europhys. Lett. 34, 411 (1996).

${ }^{20} \mathrm{O}$. N. Boratav, “On recent intermittency models of turbulence," Phys. Fluids 9, 1206 (1997).

${ }^{21}$ V. L'vov and I. Procaccia, "Hydrodynamic turbulence: A 19th century problem with a challenge for the 21st century," Phys. World 9, 35 (1996) and references to the original contributions of these authors therein.

${ }^{22}$ B. Dubrulle, "Intermittency in fully developed turbulence: Log-Poisson statistics and generalized scale covariance," Phys. Rev. Lett. 73, 959 (1994); Z. S. She and E. S. Waymire, "“'Quantized energy cascade and log-Poisson statistics in fully developed turbulence," ibid. 74, 262 (1995).

${ }^{23}$ G. Ruiz Chavarria, C. Baudet, R. Benzi, and S. Ciliberto, "Hierarchy of the velocity structure functions in fully developed turbulence," J. Phys. II 5, 485 (1995).

${ }^{24}$ A. N. Kolmogorov, "A refinement of previous hypotheses concerning the local structure of turbulence in a viscous incompressible fluid at high Reynolds number,' J. Fluid Mech. 13, 82 (1962); G. Stolovitzky, P. Kailasnath, and K. R. Sreenivasan, "Kolmogorov's refined similarity hypotheses," Phys. Rev. Lett. 69, 1178 (1992); G. Stolovitzky and K. R. Sreenivasan, "RMP Colloquium: Kolmogorov's refined similarity hypotheses for turbulence and general stochastic processes," Rev. Mod. Phys. 66, 229 (1994); S. Chen, G. D. Doolen, R. H. Kraichnan, and L. P. Wang, "Is the Kolmogorov refined similarity relation dynamic or kinematic?," Phys. Rev. Lett. 74, 1755 (1995); P. P. Wang, S. Chen, J. Brasseur, and J. C. Wyngaard, "Examination of hypotheses in the Kolmogorov refined turbulence theory through high-resolution simulations. Part 1. Velocity field,' J. Fluid Mech. 309, 113 (1996); K. R. Sreenivasan and R. A. Antonia, "The phenomenology of small-scale turbulence," Annu. Rev. Fluid Mech. 29, 435 (1997).

${ }^{25}$ J. Eggers and S. Grossmann, "Does deterministic chaos imply intermittency in fully developed turbulence?," Phys. Fluids A 3, 1958 (1991).

${ }^{26}$ S. Grossmann and D. Lohse, "Intermittency in the Navier-Stokes dynamics,"' Z. Phys. B 89, 11 (1992).

${ }^{27} \mathrm{~S}$. Grossmann and D. Lohse, "Scale resolved intermittency in turbulence,"' Phys. Rev. E 6, 611 (1994); “Universality in fully developed turbulence,"' Phys. Rev. E 50, 2784 (1994).

${ }^{28}$ J. Jimenez, A. Wray, P. G. Saffman, and R. S. Rogallo, "The structure of intense vorticity in isotropic turbulence,", J. Fluid Mech. 255, 65 (1993).

${ }^{29} \mathrm{G}$. K. Batchelor, The Theory of Homogeneous Turbulence (Cambridge University Press, Cambridge, 1953). 
${ }^{30}$ R. Benzi, S. Ciliberto, C. Baudet, and G. R. Chavarria, "On the scaling of three-dimensional homogeneous and isotropic turbulence,', Physica D 80, 385 (1995).

${ }^{31}$ S. Grossmann, D. Lohse, and A. Reeh, 'Developed turbulence: From full simulations to full mode reductions,' Phys. Rev. Lett. 77, 5369 (1996).

${ }^{32}$ R. Kerr, "Higher-order derivative correlations and the alignment of smallscale structures in isotropic numerical turbulence,' J. Fluid Mech. 153, 31 (1985); A. Vincent and M. Meneguzzi, "The spatial structure and statistical properties of homogeneous turbulence,' J. Fluid Mech. 225, 1 (1991).

${ }^{33}$ R. Benzi, L. Biferale, and E. Travatore, "Universal statistics of nonlinear energy transfer in turbulent models,'” Phys. Rev. Lett. 77, 3114 (1996).

${ }^{34}$ S. Grossmann, D. Lohse, and A. Reeh, "Spectra and structure functions in the REWA approach to high Re turbulence," in Dynamical Systems and
Chaos, Vol. 2: Physics, edited by S. Saito, K. Shiraiwa, and Y. Aizawa (World Scientific, Singapore, 1995), p. 209.

${ }^{35} \mathrm{C}$. Uhlig and J. Eggers, "Local coupling of shell models leads to anomalous scaling,', Z. Phys. B 102, 513 (1997).

${ }^{36}$ R. Benzi, R. Tripiccone, F. Massaioli, S. Succi, and S. Ciliberto, "On the scaling of the velocity and temperature structure functions in turbulent Rayleigh Benard convection,', Europhys. Lett. 25, 341 (1994).

${ }^{37}$ R. Grauer, J. Krug, and C. Marliani, "Scaling of high-order structure functions in magnetohydrodynamic turbulence,', Phys. Lett. A 195, 335 (1994).

${ }^{38}$ V. L'vov, E. Podivilov, and I. Procaccia, 'Invariants for correlations of velocity differences in turbulent fields,' Phys. Rev. Lett. 79, 2050 (1997).

${ }^{39}$ S. Grossmann, D. Lohse, and A. Reeh, "Scaling of the irreducible SO(3)invariants of velocity correlations in turbulence," preprint, submitted to Phys. Rev. 